\title{
Stochastic Travelling Advisor Problem Simulation with a Case Study: A Novel Binary Gaining-Sharing Knowledge-Based Optimization Algorithm
}

\author{
Said Ali Hassan, ${ }^{1}$ Yousra Mohamed Ayman, ${ }^{1}$ Khalid Alnowibet, ${ }^{2}$ Prachi Agrawal, ${ }^{3}$ \\ and Ali Wagdy Mohamed $\mathbb{1}^{4,5}$ \\ ${ }^{1}$ Department of Operations Research and Decision Support, Faculty of Computers and Artificial Intelligence, Cairo University, \\ Giza, Egypt \\ ${ }^{2}$ College of Science, Department of Statistics and Operations Research, King Saud University, Riyadh, Saudi Arabia \\ ${ }^{3}$ Department of Mathematics and Scientific Computing, National Institute of Technology Hamirpur, Hamirpur, \\ Himachal Pradesh 177005, India \\ ${ }^{4}$ Operations Research Department, Faculty of Graduate Studies for Statistical Research, Cairo University, Giza 12613, Egypt \\ ${ }^{5}$ Wireless Intelligent Networks Center (WINC), School of Engineering and Applied Sciences, Nile University, Giza, Egypt
}

Correspondence should be addressed to Ali Wagdy Mohamed; aliwagdy@gmail.com

Received 18 October 2020; Accepted 31 October 2020; Published 1 December 2020

Academic Editor: Ahmed Mostafa Khalil

Copyright (c) 2020 Said Ali Hassan et al. This is an open access article distributed under the Creative Commons Attribution License, which permits unrestricted use, distribution, and reproduction in any medium, provided the original work is properly cited.

\begin{abstract}
This article proposes a new problem which is called the Stochastic Travelling Advisor Problem (STAP) in network optimization, and it is defined for an advisory group who wants to choose a subset of candidate workplaces comprising the most profitable route within the time limit of day working hours. A nonlinear binary mathematical model is formulated and a real application case study in the occupational health and safety field is presented. The problem has a stochastic nature in travelling and advising times since the deterministic models are not appropriate for such real-life problems. The STAP is handled by proposing suitable probability distributions for the time parameters and simulating the problem under such conditions. Many application problems like this one are formulated as nonlinear binary programming models which are hard to be solved using exact algorithms especially in large dimensions. A novel binary version of the recently developed gaining-sharing knowledge-based optimization algorithm (GSK) to solve binary optimization problems is given. GSK algorithm is based on the concept of how humans acquire and share knowledge during their life span. The binary version of GSK (BGSK) depends mainly on two stages that enable BGSK for exploring and exploitation of the search space efficiently and effectively to solve problems in binary space. The generated simulation runs of the example are solved using the BGSK, and the output histograms and the best-fitted distributions for the total profit and for the route length are obtained.
\end{abstract}

\section{Introduction}

A new problem which we are going to call the Travelling Advisor Problem (TAP) in network optimization is defined for an advisor who wants to settle on the foremost profitable route for visiting some or all candidate workplaces each associated with a corresponding profit. He begins from a predetermined starting location and next wants to visit each chosen workplace exactly once within the day working hours. The target of the TAP is to select the route with maximum profitability for the advisory company.

The Travelling Advisor Problem is a new and different version of the well-known Travelling Salesman Problem (TSP) studied in operations research and theoretical computer science. The general idea of TSP is defined for a list of cities (represented as nodes) and their pairwise distances, and the task is to find the shortest possible tour that starts at the hometown, visits each city exactly once, and returns back 
home. Droste [1] stated that the number of different tours is very large, so one might not think to solve the problem by simply calculating the length of each possible tour. Therefore, suitable algorithms are needed to solve these situations.

Instead, in the Travelling Advisor Problem (TAP), nodes represent a set of workplaces and an advisor's job is to visit a subset of the workplaces and spend some time in each for giving his advice. In quite a nutshell, an advisor starts his working day from his company's headquarter, and then he proceeds to travel to and advise a chosen workplace and then transfers to a new one until his working hours are over. During a workday of maximum eight hours, each travelling time between one workplace and the next in addition to the advising time spent at each visited workplace is considered in addition to the travelling time spent from the headquarter to the first visited workplace. Each workplace will yield a different profit than the other, meaning that the goal is to maximize the advisor's total profit.

TAP differs from TSP in the following main points:

(i) In the TSP, the time is open till completing visiting of all customers, while in the TAP, the available time is limited by the day working hours

(ii) In the TSP, the salesman will visit all the customers, while in the TAP, the advisor will determine a route containing some or all the workplaces which optimizes the problem objective function within the available limited day time

(iii) In the TSP, no time is consumed in customer places, or it is immaterial, while in the TAP, the advising time at a workplace is a basic factor in the day time limit constraint, and hence, it directly affects the choice of the optimum solution of the problem

(iv) In the TSP, the objective is to complete the route while minimizing the total travelling time, while in the TAP the objective function is to maximize the total profit in the chosen route

In simple practical application problems where variations in travelling times and advising times are not so effective or the decision maker wants only to have a rough picture of the considered problem, these parameters can be considered as deterministic quantities. In general practice, the travelling and advising times are random and very difficult to be exactly measured or evaluated, considering that both the travelling and advising times should be independent and continuously distributed random variables.

The stochastic version of the Travelling Advisor Problem has a wide range of real-life applications in various service and advisory fields. Such fields show up in many consulting domains such as health and safety, industry, agriculture, business, education, telecommunications, investing, quality assurance, social and community services, pollution, medical, tourism, marketing, sales, advertising, sports, arts, and cooking.

The rest of this paper is organised as follows: Section 2 describes in detail the mathematical model of the Travelling Advisor Problem (TAP) including the definition of problem variables, constraints, and the objective function. The proposed model is a nonlinear binary model with a dimension depending on the number of candidate workplaces.

The Stochastic Travelling Advisor Problem (STAP) simulation procedure is explained in detail in Section 3. The new problem proposes PERT-beta probability distributions for the travelling and advising times and resolving the problem under such conditions, and the steps of the simulation solution procedure are also explained.

A real practical application case study is explained in Section 4; the case study is implemented in a very important service sector which is the occupational health and safety. This section reveals also the role and advices offered by the Occupational and Health Advisor to organizations and workplaces.

In Section 5, a novel binary version of a recently developed gaining-sharing knowledge-based optimization algorithm (GSK) is introduced to solve the TAP. GSK cannot solve the problem with binary space; therefore, binary gaining-sharing knowledge-based optimization algorithm (BGSK) is proposed with two new binary junior and senior stages. These stages allow BGSK to explore and exploit the search space of the problem efficiently; an example of the experimental results of one of the simulation runs of STAP is also presented. Section 6 gives the conclusions and the suggested points for future research studies, respectively.

\section{Mathematical Model for the Travelling Advisor Problem (TAP)}

The new Travelling Advisor Problem (TAP) is defined on a graph $G$ with a set of $n$ nodes $V$ representing the workplaces (customers) and an additional node denoting the Health and Safety Agency headquarter (HQ) where the advisor starts the job and a set of arcs representing the travelling times between two distinct workplaces (Pinter [2]). The time of inspection in a workplace and the travelling time between two workplaces are specified.

While the advisor should start his route at the headquarter (HQ), he ends his route at the last visited workplace.

The mathematical model for such a deterministic TAP with known and fixed parameters representing the travelling and advising times is formulated as follows.

\subsection{Decision Variables. Let}

$x_{i}^{m}= \begin{cases}1, & \text { if workplace } i \text { is visited by the advisor on position } m \text { of his route, } i \text { and } m=1,2, \ldots, n=\text { total number of workplaces, } \\ 0, & \text { otherwise. }\end{cases}$ 


\subsection{Constraints}

2.2.1. Position Constraints. Each position $m$ in the advisor route has at most one workplace:

$$
\sum_{i=1}^{n} x_{i}^{m} \leq 1, \quad m=1,2, \ldots, n .
$$

2.2.2. Workplace Constraints. Each workplace $i$ can be in one position of the advisor route or not visited:

$$
\sum_{m=1}^{n} x_{i}^{m} \leq 1, \quad i=1,2, \ldots, n .
$$

2.2.3. Consecutive Position Constraints. A position $(m+1)$ cannot exist in the advisor tour unless the preceding position $m$ exists, and this is achieved by the following set of constraints:

$$
\sum_{i=1}^{n} x_{i}^{m+1} \leq \sum_{i=1}^{n} x_{i}^{m}, \quad m=1,2, \ldots, n-1 .
$$

(i) If $\sum_{i=1}^{n} x_{i}^{m+1}=1$, then $\sum_{i=1}^{n} x_{i}^{m}=1, m=1,2, \ldots, n-1$

(ii) If $\sum_{i=1}^{n} x_{i}^{m+1}=0$, then there is no restriction on the value of $\sum_{i=1}^{n} x_{i}^{m}=1, m=1,2, \ldots, n-1$

2.2.4. Working Hours/Day Constraints. The total time spent by the advisor in travelling and site inspection should be within the maximum working hours/day $=T=8$ hours:

$$
\sum_{i=1}^{n} t_{0, i} x_{i}^{1}+\sum_{m=1}^{n} \sum_{i=1}^{n}\left(t_{i} x_{i}^{m}\right)+\sum_{i=1}^{n} \sum_{\substack{j=1 \\ j \neq i}}^{n} t_{i, j} \cdot\left(\sum_{m=1}^{n-1} x_{i}^{m} \cdot x_{j}^{m+1}\right) \leq T,
$$

where

(i) $t_{0, i}=$ transportation time between the headquarter and workplace $i$, where $i=1,2, \ldots, n$

(ii) $t_{i, j}=$ transportation time between the two adjacent workplaces $i$ and $j$, where $i, j=1,2, \ldots, n$

(iii) $t_{i}=$ inspection time for workplace $i$, where $i=1,2$, $\ldots, n$

This is a quadratic inequality in two variables, the first part is for travelling from the headquarter to the first position in the route, and the second part is the inspection times at workplaces, and the third part is the travelling time between different positions in the route.

2.2.5. Binary Constraints. All the decision variables are $0-1$ :

$$
x_{i}^{m}=0 \text { or } 1, i, m=1,2, \ldots, n .
$$

2.2.6. The Objective Function. It is formulated for maximizing the total profits of the Occupational Health and Safety Agency gained by visiting the workplaces during the working day time limit:

$$
\operatorname{Max} z=\sum_{m=1}^{n} \sum_{i=1}^{n} p_{i} x_{i}^{m}
$$

in which $p_{i}=$ profit of visiting workplace $i$, where $i=1,2, \ldots$, $n$.

The optimum solution will produce two distinct situations:

(1) If $\sum_{m=1}^{n} x_{i}^{m}=1 i=1,2, \ldots, n$, then all the $n$-workplaces are visited by the advisor in one working day and the problem is completed.

(2) If $\sum_{m=1}^{n} x_{i}^{m}=0$ for any $\mathrm{i}$, then the corresponding workplace $i$ is not visited by the advisor in the first working day. In this case, it is needed to eliminate the visited workplaces, adding one more day and repeat the procedure for another working day.

\section{Stochastic Travelling Advisor Problem Simulation Procedure}

The problem so far has been concerned with deterministic travelling and advising times. However, solutions of the deterministic models deteriorate once applied in real-life problems where these times are stochastic [3]. In this section, the Stochastic Travelling Advisor Problem (STAP) will be handled, by proposing probability distributions for the time constraints and resolving the problem under such conditions.

The probability distribution function (PDF) used for time simulations should be continuous and limited between two-time intercepts and have a unique mode in its defined range therefore making it ideal to choose here, as the beta PDF satisfies all the conditions stated [4]. Probability distribution of travelling times using beta distribution has been proposed and validated by many researchers [5]. The beta distribution is very flexible and commonly used to represent where the uncertain variable is a random value between zero and a positive value [6]. The beta distribution is flexibly described over the interval $[0,1]$ and the beta density function is a very flexible form to represent outcomes like probabilities [7]. Applications include modelling random variables that have a finite range $a$ to $b$. The most famous application is the distribution of activity times in project networks like that of the advising time in STAP [8].

The probability density function of beta distribution is given by

$$
\operatorname{prob}(x \mid \alpha t, n \beta)=\frac{x^{\alpha-1} *(1-x)^{\beta-1}}{B(\alpha, \beta)},
$$

where the beta function is given by

$$
\operatorname{Beta}(\alpha, \beta)=\int_{0}^{1} t^{\alpha-1}(1-t)^{\beta-1} \mathrm{~d} t
$$


Alpha $(\alpha)$ and beta $(\beta)$ can be any positive value greater than zero. They are called the shape parameters of the beta distribution [6]. These two parameters work together to determine whether the distribution is symmetrical, positively skewed, or negatively skewed. Depending on the values of $\alpha$ and $\beta$, the beta distribution can take a variety of shapes [9]. There are many graph packages that allow to experiment with different values of $\alpha$ and $\beta$ and visualize how the shape changes (see, for example, Rosenmai [10]).

In PERT method, three-point estimate for activity duration is an estimate that includes optimistic, most likely, and pessimistic estimate. This method is known as program evaluation and review technique (PERT) analysis or PERT method [11]. The PERT distribution is a family of continuous probability distributions defined by the minimum or best $(a)$, most likely $(m)$, and maximum or worst $(b)$ values that a variable can take. It is a transformation of the four-parameter beta distribution with an additional assumption on its expected value.

PERT introduces uncertainty into the estimates for activity duration and is well suited for those situations where there is insufficient background information to specify accurately time. Its three-time estimates become the framework on which the probability distribution curve for the activity is erected [12]. Therefore, PERT has become a classic tool for estimating uncertain activities durations [13].

The three estimates of the activity duration enable the expected mean time $\mu$, as well as the standard deviation $\sigma$ and variance $\sigma^{2}$ to be derived mathematically (Shankar et al. [14] and Golpîra [15]):

$$
\begin{aligned}
\mu & =\frac{1}{6}(a+4 m+b), \\
\sigma^{2} & =\frac{1}{36}(b-a)^{2} .
\end{aligned}
$$

The PDF of PERT distribution can be symmetric, rightskewed, or left-skewed according to the values of the parameters $a, m$, and $b$ [16]. This is better clarified with visual illustrations of the PERT Distribution using special computer programs (for example, Rosenmai [10] and EasyFit [17]).

3.1. PERT-Beta Distributions. The built-in beta distributions provided by most software systems are parameterized by two shape parameters $(\alpha$ and $\beta$ ) and two location parameters $(a$ and $b$ ). In the PERT context, the desired beta distributions are specified by two statistics (mean and variance) and the same two location parameters $(a$ and $b$ ). Hence, in order to carry out simulation with the software, one needs to first convert the PERT mean and variance and the extreme values $a$ and $b$ into the associated shape parameters $\alpha$ and $\beta$ [18]. When these PERT mean and variance formulas are substituted into beta formulas, one gets the unique beta distribution parameters $\alpha$ and $\beta$ needed for each time activity as a beta distribution which varies between $(a$ and $b$ ) and fits for modelling the activity duration [13].
Beta distributions defined on the interval $[a, b]$ in this fashion are referred as PERT-beta distributions because they exhibit means and variances as specified by the PERT mean and variance formulas. PERT-beta distributions have the following formulas for location parameters ( $a$ and $b$ ) and shape parameters $(\alpha$ and $\beta)$ [19]:

$$
\begin{aligned}
& \alpha=\left(\frac{\mu-a}{b-a}\right)\left[\left(\frac{(\mu-a)(b-\mu)}{\sigma^{2}}\right)-1\right], \\
& \beta=\left(\frac{b-\mu}{b-a}\right)\left[\left(\frac{(\mu-a)(b-\mu)}{\sigma^{2}}\right)-1\right] .
\end{aligned}
$$

The best, most likely, and worst travelling times are estimated based on experience and with the help of Google Maps to get traffic data from the starting position to each candidate workplace and from each workplace to another in any given day and time. The minimum, most likely, and maximum advising times are also estimated based on the experience of the advising group.

PERT-beta distribution is used to generate random values for the travelling and advising times using the $\operatorname{BETAINV}(R A N D, \alpha, \beta, a, b)$ function [19]. In Excel, the $\mathrm{RAND}()$ function is used to obtain uniformly distributed probability values. The generated random values for the travelling and advising times are introduced into the mathematical model, the model is solved using an appropriate method, and the results are gathered including the optimum solution, the route duration, and the expected total profit. The process of generating other random variables is repeated many times while the corresponding run results are gathered in each time. After enough number of runs, the final concluded results are worked out. The steps of the solution procedure are shown in Figure 1.

\section{Practical Application Case Study}

One of the most important areas of the service sector is chosen in this case study, which is the occupational health and safety field.

Workers in all countries around the world are exposed to many hazards while working in their workplaces [20]. Among these hazards are the chemical hazards [21] and the biological hazards [22].

The purpose of health and safety advisor is to facilitate and promote safety, health, and wellness across all function areas of organizations, to collaborate with both staff and management to ensure regulatory compliance and to undertake reviewing, audits, and developing strategies for best practices [23].

Occupational Health and Safety (OH\&S) Acts and Regulations are laws that govern workplaces. They outline the rights and responsibilities of the employer, the worker, and the supervisor to ensure working environments are healthy and safe [24]. Egyptian law regulations in this field are enrolled under the International Labour Organization and the World Health Organization (WHO) $[25,26]$.

It is the responsibility of the advising agency to check up the risks at the workplace and those that can happen, and 


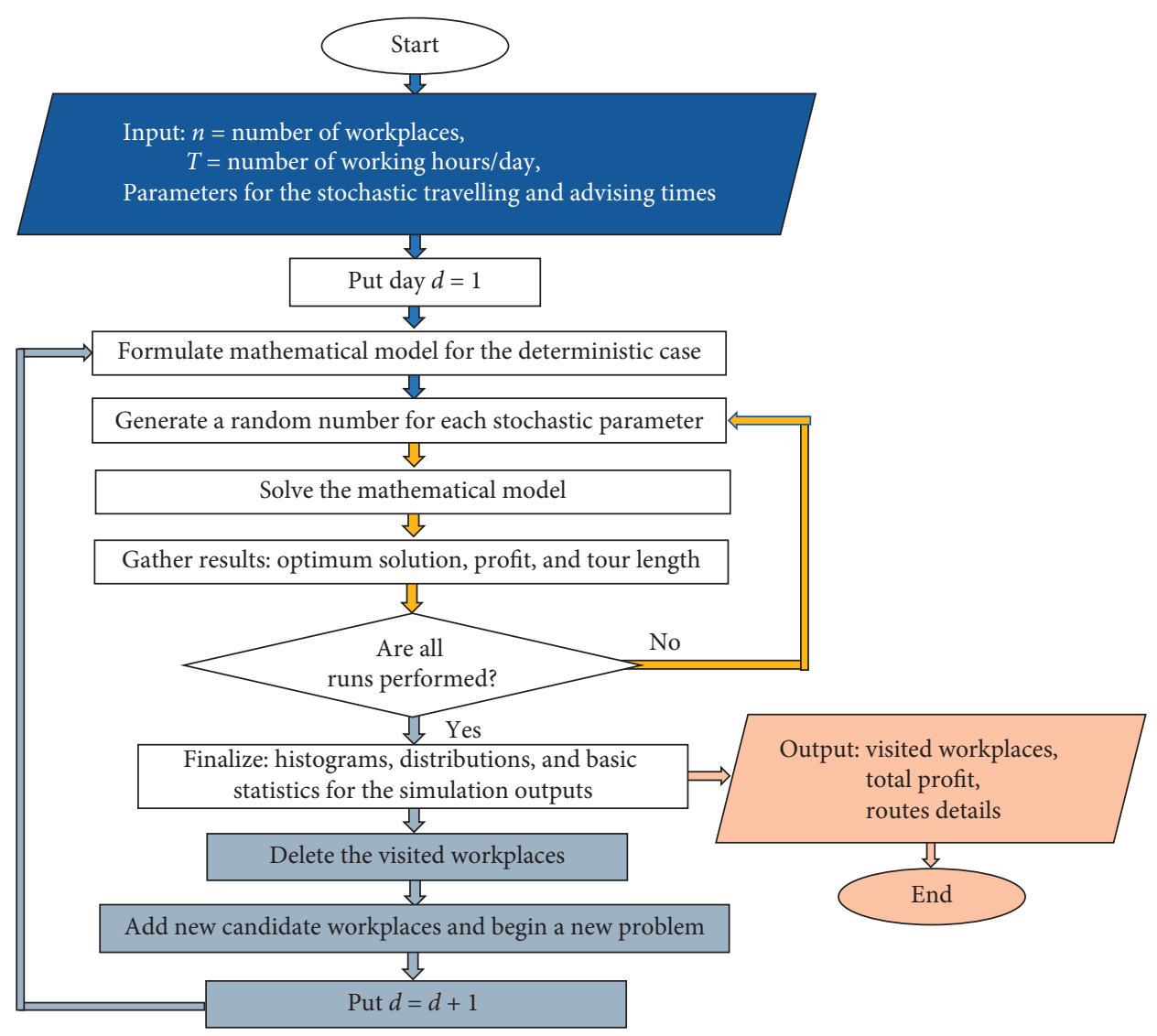

Figure 1: Steps of the simulation procedure of the stochastic TAP.

these inspections help distinguishing and preventing risk [27]. Health and safety advisors make sure that people comply with all rules of safety and health and that work environments are not the source of illness, wounding, or dying [28].

Advisors accomplish their work by visiting workplaces to ensure that work is accurately performed in compliance with regulations, Marriott et al. [29]. Other responsibilities are to predict possible hazards, providing advices on health and safety, recording violations of regulations, gathering illegal evidence, follow-up evidence in court, writing remarks and reports, and providing training and educational support to employers [30].

The Faculty of Engineering, Cairo University, located in Giza, Giza Governorate, is chosen as the starting position where the advising group will start the route as one of its research centers is responsible for this advising project. The five factories shown in Table 1 are the candidate workplaces to be visited, and the table shows also the profit for the visit of each workplace. Using a Google Maps tool "My Map," a location map is created to pinpoint the factory locations for better illustration as shown in Figure 2.

4.1. Estimating the Probabilistic Times. The minimum (best), most likely, and maximum (worst) travelling times are calculated using Google Maps and past practical experience to get traffic data from the starting location to each workplace and from each workplace to another. Figure 3 shows an illustrative example for estimating the parameters ( $a$ and $b$ ) for the travelling time from the starting location to Workplace number 3 (Eastern Company). The most likely time $m$ is estimated using experience, and it is in the interval [ $a$ and $b$ ] and depends mainly on the chosen day of the week and time of the day. In the same manner, other time parameters are estimated for all the travelling times of the case study. Table 2 shows the PERT-beta distribution parameters of the travelling times from the starting location to the workplaces.

Similarly, the parameters $(a, m$, and $b)$ for PERT distribution of the advising time at each workplace are estimated based on the experience of the advisory group. Table 3 represents the PERT-beta distribution parameters of the advising times. Figure 4 expresses some examples of the beta $\mathrm{BDF}$ and CDF for travelling and advising times [31]. The distributions are right-skewed for all the five stochastic advising times, as $m$ values are closer to the $a$ values than they are to the $b$ values.

Using such PERT-beta distributions, 30 simulation random values are generated using Excel software for all the travelling and advising times. An example of the generated values for the starting travelling and for the advising times is shown in Table 4 and 10 examples of the generated travelling times between workplaces are shown in Table 5 .

The simulation process is repeated 30 times, the generated random values for the used parameters according to 
TABLE 1: Workplaces for the case study.

\begin{tabular}{cccc}
\hline$\#$ & Factory & Address & $\begin{array}{c}\text { Profit } \\
(\$) \times(102)\end{array}$ \\
\hline 1 & El Nasr Co. For Intermediate & $\mathrm{Km} \mathrm{28,} \mathrm{Cairo} \mathrm{Alex} \mathrm{Desert} \mathrm{Rd.,} \mathrm{Industrial} \mathrm{Zone,} \mathrm{Abou-Rawash,} \mathrm{Giza.} \mathrm{Beside} \mathrm{Smart}$ & 3.1 \\
2 & Chemicals & Village & \\
3 & Eva Pharma & El-Sadat, Zawya Abou Muslim, Giza Governorate & 4.7 \\
4 & Eastern Company & $450 \mathrm{Al}$ Haram, Al Omraneyah Al-Sharqeyah, El-Omraniya, Giza Governorate & 5.5 \\
5 & El Helal and Golden Star & Industrial Zone A, 10th of Ramadan, Cairo Governorate & 4.2 \\
\hline
\end{tabular}

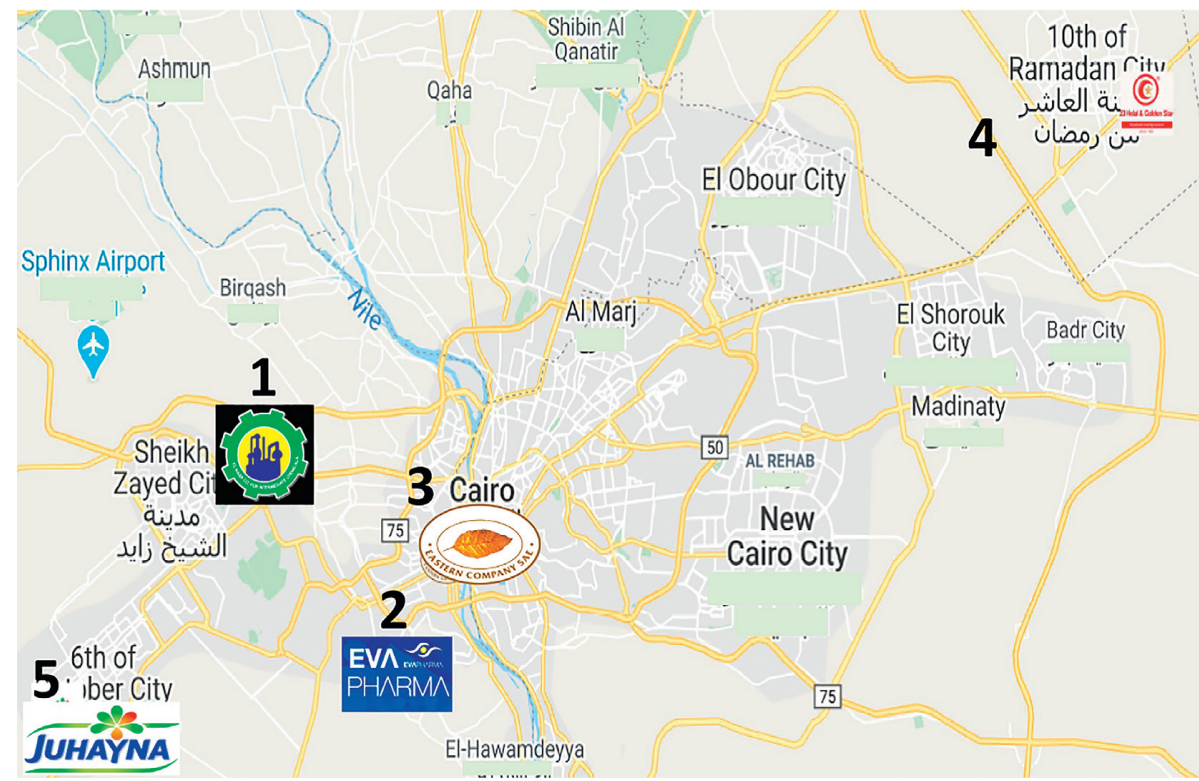

FIgURE 2: Location of the candidate workplaces.

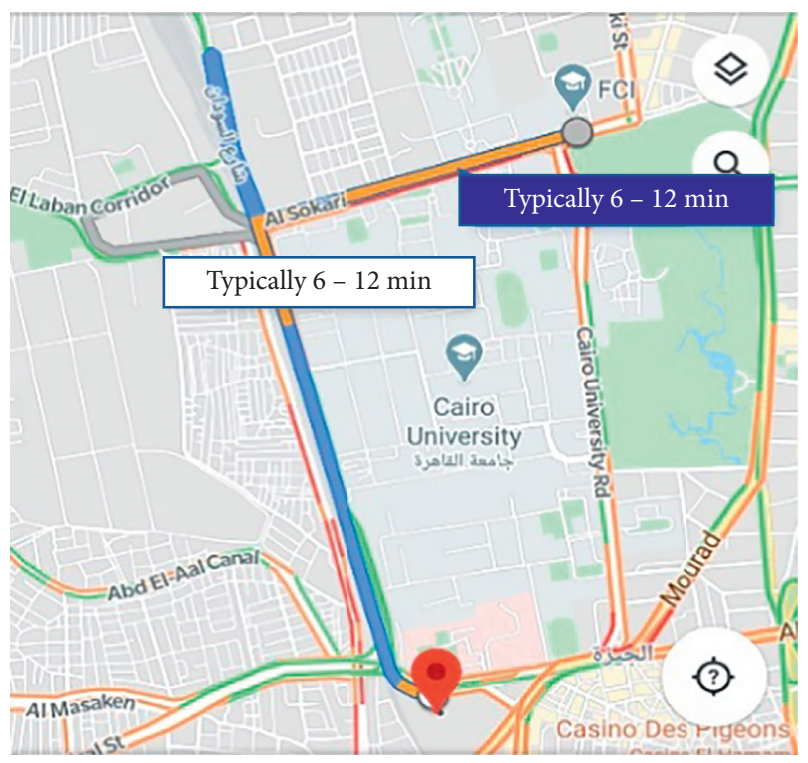

Figure 3: Parameters of the stochastic travelling time.

the BERT-beta distribution for each simulation run are substituted in the mathematical model described in Section 2 , and the optimum solution is obtained for each case for further evaluation.

\section{Proposed Methodology}

Metaheuristic algorithms have been developed to solve the complex optimization problem with continuous variables. Mohamed et al. [32] recently proposed a novel gainingsharing knowledge-based optimization algorithm (GSK), which is based on the ideology of acquiring knowledge and sharing it with others throughout their lifetime. The original GSK solves optimization problems over continuous space, but it cannot solve the problem with binary space. So, a new variant of GSK is introduced to solve the proposed TAP. A novel binary gaining-sharing knowledge-based optimization algorithm (BGSK) is proposed over discrete binary space with new binary junior and senior gaining and sharing stages.

On the other hand, there are many constraint handling techniques in the literature (Deb [33]; Cello [34]; Muangkote et al. [35]. In this paper, the augmented Lagrangian method is used to handle the constraints, in which a constrained optimization problem is converted into an unconstrained optimization problem (Long et al. [36]; Bahreininejad [37]). The proposed methodology is described as follows.

5.1. Gaining-Sharing Knowledge-Based Optimization Algorithm (GSK). A constrained optimization problem can be formulated mathematically as follows: 
TABLE 2: PERT-beta distribution parameters of the travelling times from the starting location.

\begin{tabular}{lccccccc}
\hline$t_{0, \mathbf{i}}$ & $a$ & $m$ & $b$ & $\mu$ & $\sigma$ & $\alpha$ \\
\hline$t_{0,1}$ & 22 & 35 & 45 & 3.125 & 0.208 & 3.85 \\
$t_{0,2}$ & 22 & 40 & 45 & 12 & 4.1667 & 0.15 & 4.32 \\
$t_{0,3}$ & 6 & 10 & 105 & 5.183 & 0.25 & 4.23 \\
$t_{0,4}$ & 70 & 60 & 65 & 1.575 & 0.3167 & 3.08 \\
$t_{0,5}$ & 45 & 60 & 0.092 & 3.33 \\
\hline
\end{tabular}

TABLE 3: PERT-beta distribution parameters of the advising times.

\begin{tabular}{cccccccc}
\hline$t_{i}$ & $a$ & $m$ & $b$ & $\mu$ & $\sigma$ & $\alpha$ & $\beta$ \\
\hline$t_{1}$ & 2.75 & 3 & 4 & 3.13 & 0.04 & 1.27 & 4.29 \\
$t_{2}$ & 1.85 & 2 & 2.75 & 2.1 & 0.02 & 1.01 & 4.22 \\
$t_{3}$ & 3.75 & 4 & 5.25 & 4.17 & 0.06 & 1.01 & 4.22 \\
$t_{4}$ & 4.6 & 5 & 6.5 & 5.18 & 0.1 & 1.35 & 4.31 \\
$t_{5}$ & 1.45 & 1.5 & 2 & 1.58 & 0.01 & 0.44 & 3.89 \\
\hline
\end{tabular}

$$
\begin{array}{ll}
\text { Min } & f(X) ; X=\left[x_{1}, x_{2}, \ldots, x_{\text {Dim }}\right], \\
\text { s.to. } & g_{i}(X) \leq 0 ; i=1,2, \ldots, m, \\
& X \in\left[\alpha_{p}, \beta_{p}\right] ; p=1,2, \ldots, \operatorname{Dim},
\end{array}
$$

where $f$ denotes the objective function; $X=\left[x_{1}, x_{2}, \ldots, x_{\text {Dim }}\right]$ are the decision variables; $g_{i}(X)$ are the inequality constraints, and $\alpha_{p}$ and $\beta_{p}$ are the lower and upper bounds of decision variables, respectively, and Dim represents the dimension of individuals. If the problem is in maximization form, then consider minimization $=-$ maximization .

The human-based algorithm GSK is of two stages: junior and senior gaining and sharing stages. All persons acquire knowledge and share their views with others. The people from early-stage gain knowledge from their small networks such as family members, relatives, and neighbours want to share their opinions with others who might not be from their networks, due to curiosity of exploring others. These people may not have the experience to categorize the people. In the same way, the people from the middle or later age enhance their knowledge by interacting with friends, colleagues, social media friends, etc., and share their views with the most suitable person, so that they can improve their knowledge. These people have the experience to judge other people and can categorize them (good or bad). The process mentioned above can be formulated mathematically in the following steps:

Step 1: to obtain the starting solution of the optimization problem, the initial population must be obtained. The initial population is created randomly within the boundary constraints as follows:

$$
x_{t p}^{0}=\alpha_{p}+\operatorname{rand}_{p}\left(\beta_{p}-\alpha_{p}\right)
$$

where $t$ is for the number of populations and $\operatorname{rand}_{p}$ denotes uniformly distributed random number between 0 and 1 .

Step 2: at the beginning, the dimensions of the junior and senior stages should be computed through the following formula:

$$
\begin{aligned}
\operatorname{Dim}_{J} & =\operatorname{Dim} \times\left(\frac{\mathrm{Gen}^{\text {max }}-G}{\mathrm{Gen}^{\text {max }}}\right)^{k}, \\
\operatorname{Dim}_{S} & =\operatorname{Dim}-\operatorname{Dim}_{J},
\end{aligned}
$$

where $k(>0)$ denotes the knowledge rate that controls the experience rate, $\operatorname{Dim}_{I}$ and $\operatorname{Dim}_{S}$ represent the dimension for the junior and senior stages, respectively, $\mathrm{Gen}^{\mathrm{max}}$ is the maximum number of generations, and $G$ denotes the generation number.

Step 3: junior gaining-sharing knowledge stage: in this stage, the early aged people gain knowledge from their small networks and share their views with the other people who may or may not belong to their group. Thus, individuals are updated as follows:

According to the objective function values, the individuals are arranged in ascending order. For every $x_{t}(t=1,2, \ldots, \mathrm{NP})$, select the nearest best $\left(x_{t-1}\right)$ and worst $\left(x_{t-1}\right)$ to gain knowledge and also choose randomly $\left(x_{r}\right)$ to share knowledge. Therefore, to update the individuals, the pseudocode is presented in Figure 5.

Step 4: senior gaining-sharing knowledge stage: this stage comprises the impact and effect of other people (good or bad) on the individual. The updated individual can be determined as follows:

The individuals are classified into three categories (best, middle, and worst) after sorting individuals into ascending order (based on the objective function values). Best individual $=100 p \% \quad\left(x_{\text {best }}\right), \quad$ middle individual $=\operatorname{Dim}-2 * 100 p \% \quad\left(x_{\text {middle }}\right)$, and worst individual $=100 p \%\left(x_{\text {worst }}\right)$. For every individual $x_{t}$, choose two random vectors of the top and bottom $100 p \%$ individual for gaining part and the third one (middle individual) is chosen for the sharing part. Therefore, the new individual is updated through the pseudocode presented in Figure 6.

\subsection{Binary Gaining-Sharing Knowledge-Based Optimization} Algorithm (BGSK). To solve problems in discrete binary space, a novel binary gaining-sharing knowledge-based optimization algorithm (BGSK) was proposed. In BGSK, the new initialization and the working mechanism of both stages (junior and senior gaining-sharing stages) are introduced over binary space, and the remaining algorithms remain the same as the previous one. The working mechanism of BGSK is presented in the following sections. 


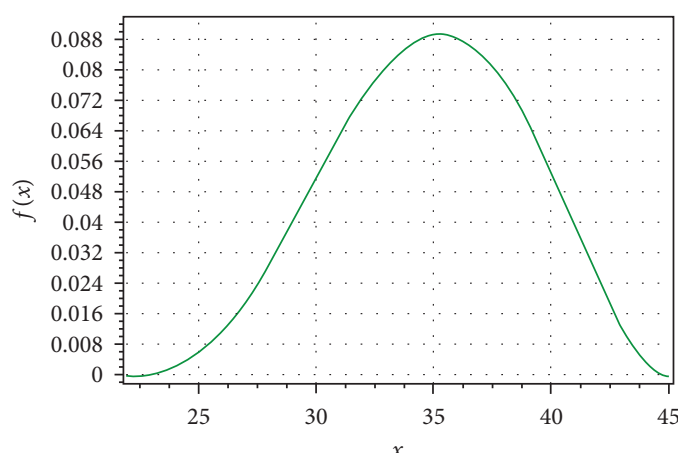

(a)

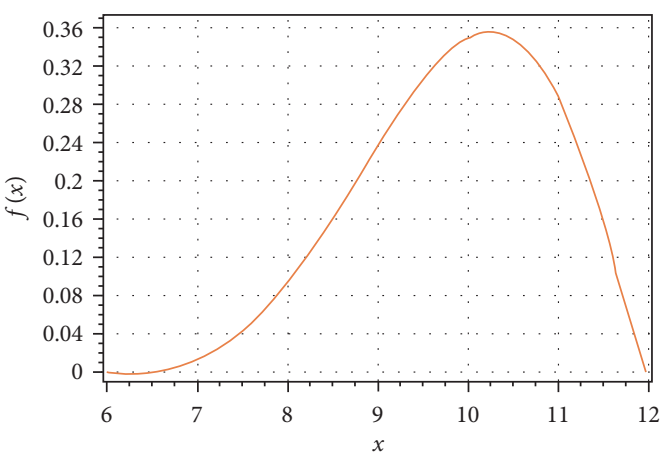

(c)

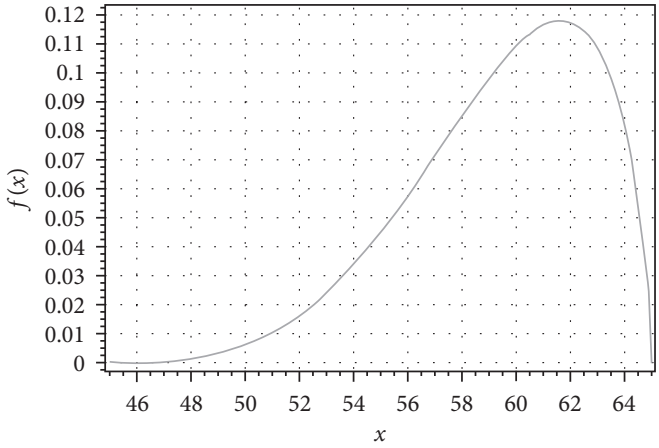

(e)

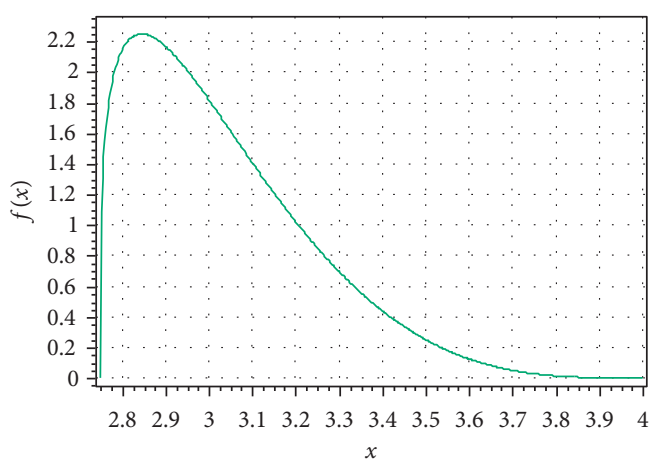

(g)

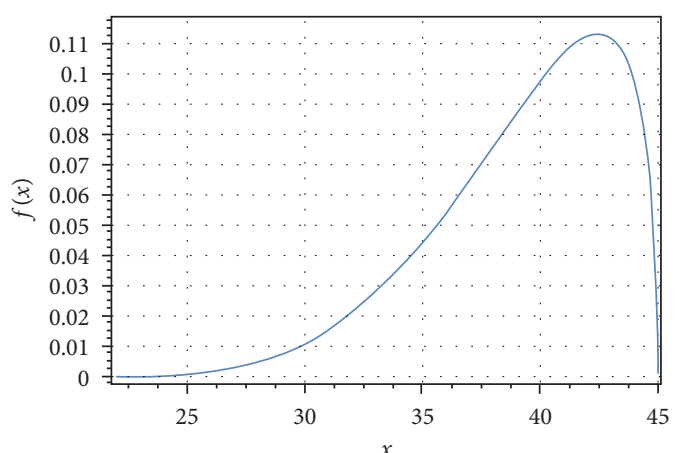

(b)

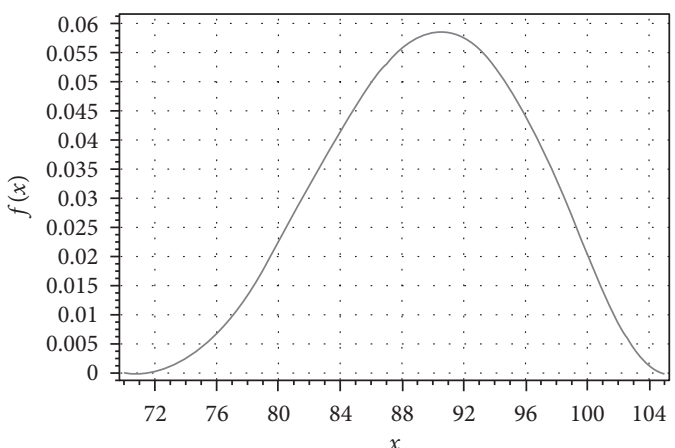

(d)

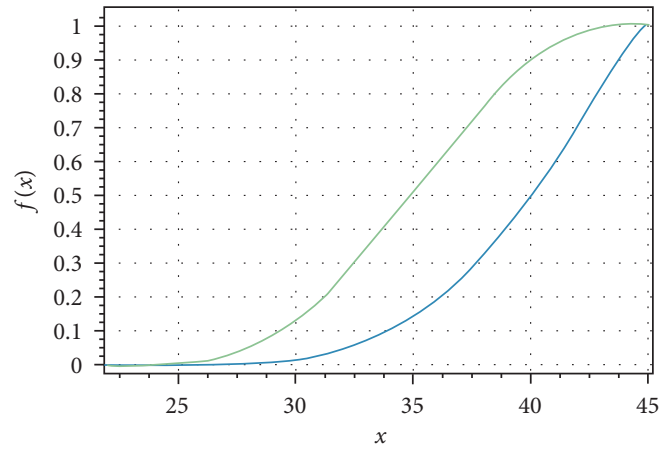

(f)

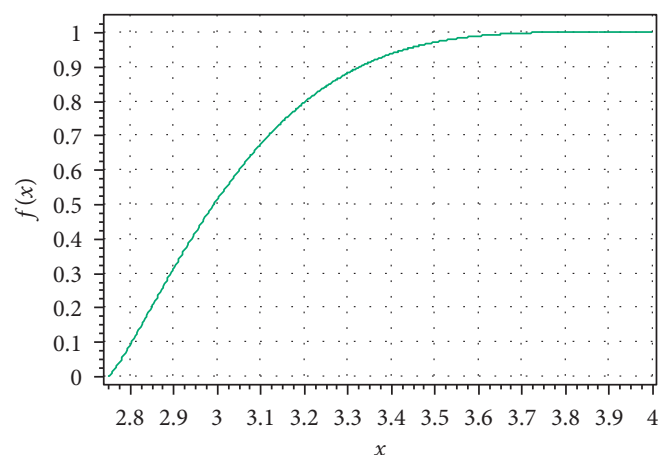

(h)

FIGURE 4: Examples of beta BDF and CDF for travelling and advising times. (a) BDF for $t_{0,1}$; (b) BDF for $t_{0,2}$; (c) BDF for $t_{0,3}$; (d) BDF for $t_{0,4}$; (e) BDF for $t_{0,5} ;$ (f) CDF for $t_{0,1}$ and $t_{0,2} ;(\mathrm{g}) \mathrm{BDF}$ for $t_{1}$; (h) CDF for $t_{1}$. 
TABLE 4: An example of the generated random values for travelling and advising times.

\begin{tabular}{lcccccc}
\hline & Workplace & \multicolumn{5}{c}{$t_{i, j}$ (hours) } \\
\hline & & 1 & 2 & 3 & 4 & 5 \\
$t_{i}$ (hours) & 0 & 0.59 & 0.62 & 0.2 & 1.61 & 1.05 \\
3.041 & 1 & & 0.78 & 0.68 & 1.58 & 1.25 \\
1.975 & 2 & & & 0.74 & 1.59 & 0.99 \\
3.87 & 3 & & & & 1.36 & 1.39 \\
4.771 & 4 & & & & & 2.2 \\
1.529 & 5 & & & & & \\
\hline
\end{tabular}

TABle 5: Examples of generated travelling times between workplaces.

\begin{tabular}{ccccccccccc}
\hline$\#$ & $t_{1,2}$ & $t_{1,3}$ & $t_{1,4}$ & $t_{1,5}$ & $t_{2,3}$ & $t_{2,4}$ & $t_{2,5}$ & $t_{3,4}$ & $t_{3,5}$ & $t_{4,5}$ \\
\hline 1 & 0.78 & 0.68 & 1.58 & 1.25 & 0.74 & 1.59 & 0.99 & 1.36 & 1.39 & 2.2 \\
2 & 0.67 & 0.64 & 1.53 & 1.15 & 0.81 & 1.23 & 0.85 & 1.56 & 1.27 & 2.60 \\
3 & 0.62 & 0.64 & 1.54 & 1.24 & 0.78 & 1.41 & 0.81 & 1.25 & 1.49 & 2.28 \\
4 & 0.81 & 0.65 & 1.66 & 1.12 & 0.8 & 1.59 & 0.82 & 1.35 & 1.44 & 2.04 \\
5 & 0.70 & 0.72 & 1.54 & 1.28 & 0.79 & 1.22 & 0.99 & 1.54 & 1.44 & 2.12 \\
6 & 0.80 & 0.71 & 1.44 & 1.21 & 0.83 & 1.39 & 0.66 & 1.48 & 1.49 & 1.86 \\
7 & 0.80 & 0.58 & 1.53 & 1.30 & 0.75 & 1.35 & 0.91 & 1.42 & 1.48 & 2.33 \\
8 & 0.72 & 0.67 & 1.61 & 1.30 & 0.75 & 1.46 & 0.89 & 1.63 & 1.35 & 2.15 \\
9 & 0.68 & 0.75 & 1.49 & 1.25 & 0.82 & 1.32 & 0.97 & 1.35 & 1.38 & 2.49 \\
10 & 0.76 & 0.74 & 1.56 & 1.19 & 0.82 & 1.60 & 0.97 & 1.49 & 1.43 & 2.19 \\
\hline
\end{tabular}

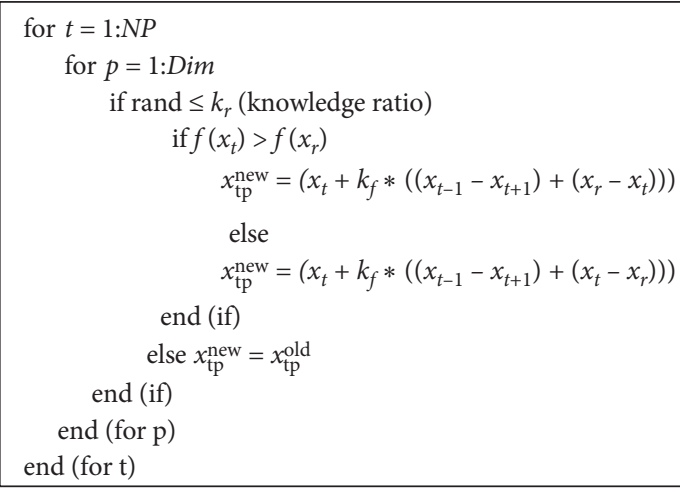

FIGURE 5: Pseudocode for junior gaining-sharing knowledge stage. Note: $k_{f}(>0)$ is the knowledge factor.

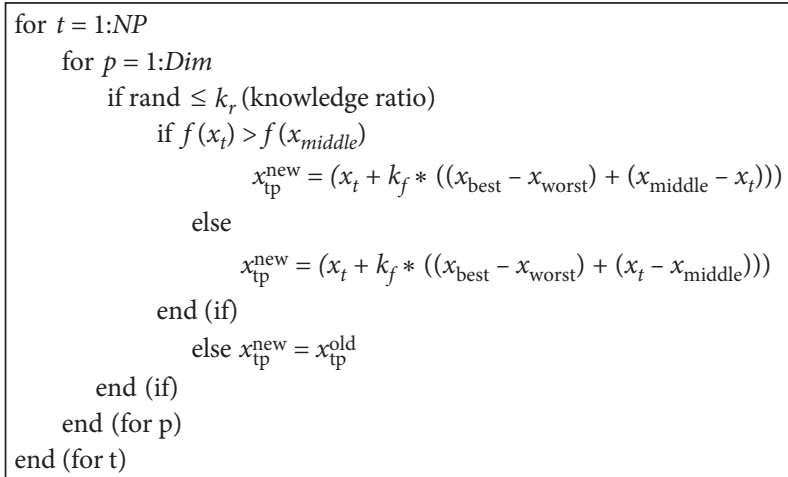

Figure 6: Pseudocode for senior gaining-sharing knowledge stage. Note: $p \in[0,1]$ is the percentage of best and worst classes.
5.2.1. Binary Initialization. The initial population is obtained in GSK using equation (18) and it must be updated using the following equation for binary population:

$$
x_{t p}^{0}=\operatorname{round}(\operatorname{rand}(0,1)) \text {, }
$$

where the round operator is used to convert the decimal number into the nearest binary number.

5.2.2. Binary Junior Gaining and Sharing Stage. The binary junior gaining and sharing stage is based on the original GSK with $k_{f}=1$. The individuals are updated in original GSK using the pseudocode (Figure 2) which contains two cases. These two cases are defined for binary stage as follows:

Case 1. When $f\left(x_{r}\right)<f\left(x_{t}\right)$ : there are three different vectors $\left(x_{t-1}, x_{t+1}, x_{r}\right)$, which can take only two values (0 and 1$)$. Therefore, a total of $2^{3}$ combinations are possible, which are listed in Table 3. Furthermore, these eight combinations can be categorized into two different subcases ((a) and (b)) and each subcase has four combinations. The results of each possible combination are presented in Table 6.

Subcase (a): if $x_{t-1}$ is equal to $x_{t+1}$, the result is equal to $x_{r}$

Subcase (b): when $x_{t-1}$ is not equal to $x_{t+1}$, then the result is the same as $x_{t-1}$ by taking -1 as 0 and 2 as 1

The mathematical formulation of Case 1 is as follows:

$$
x_{t p}^{\text {new }}= \begin{cases}x_{r} ; & \text { if } x_{t-1}=x_{t+1} \\ x_{t-1} ; & \text { if } x_{t-1} \neq x_{t+1} .\end{cases}
$$

Case 2. When $f\left(x_{r}\right) \geq f\left(x_{t}\right)$ : there are four different vectors $\left(x_{t-1}, x_{t}, x_{t+1}, x_{r}\right)$, that consider only two values ( 0 and 1$)$. Thus, there are $2^{4}$ possible combinations that are presented in Table 7. Moreover, the 16 combinations can be divided into two subcases ((c) and (d)). Subcases (c) and (d) have four and twelve combinations, respectively.

Subcase (c): if $x_{t-1}$ is not equal to $x_{t+1}$, but $x_{t+1}$ is equal to $x_{r}$, the result is equal to $x_{t-1}$

Subcase (d): if any of the conditions arise $x_{t-1}=x_{t+1} \neq x_{r}$ or $x_{t-1} \neq x_{t+1} \neq x_{r}$ or $x_{t-1}=x_{t+1}=x_{r}$, the result is equal to $x_{t}$ by considering -1 and -2 as 0 , and 2 and 3 as 1

The mathematical formulation of Case 2 is as follows:

$$
x_{t p}^{\text {new }}= \begin{cases}x_{t-1} ; & \text { if } x_{t-1} \neq x_{t+1}=x_{r} \\ x_{t} ; & \text { otherwise }\end{cases}
$$

5.2.3. Binary Senior Gaining and Sharing Stage. The working mechanism of binary senior gaining and sharing stage is the same as the binary junior gaining and sharing stage with value of $k_{f}=1$. The individuals are updated in the original senior gaining-sharing stage using pseudocode (Figure 3) that contains two cases. The two cases were further modified 
TABLE 6: Results of the binary junior gaining and sharing stage of Case 1 with $k_{f}=1$.

\begin{tabular}{lccccc}
\hline & $x_{t-1}$ & $x_{t+1}$ & $x_{r}$ & Results & Modified results \\
\hline \multirow{3}{*}{ Subcase (a) } & 0 & 0 & 0 & 0 & 0 \\
& 0 & 0 & 1 & 1 & 1 \\
& 1 & 1 & 0 & 0 & 0 \\
& 1 & 1 & 1 & 1 & 1 \\
\hline \multirow{3}{*}{ Subcase (b) } & 1 & 0 & 0 & 1 & 1 \\
& 1 & 0 & 1 & 2 & 1 \\
& 0 & 1 & 0 & -1 & 0 \\
\hline
\end{tabular}

TABLE 7: Results of the binary junior gaining and sharing stage of Case 2 with $k_{f}=1$.

\begin{tabular}{lcccccc}
\hline & $x_{t-1}$ & $x_{t}$ & $x_{t+1}$ & $x_{r}$ & Results & Modified results \\
\hline \multirow{5}{*}{ Subcase (c) } & 1 & 1 & 0 & 0 & 3 & 1 \\
& 1 & 0 & 0 & 0 & 1 & 1 \\
& 0 & 1 & 1 & 1 & 0 & 0 \\
& 0 & 0 & 1 & 1 & -2 & 0 \\
\hline \multirow{5}{*}{ Subcase (d) } & 0 & 0 & 0 & 0 & 0 & 0 \\
& 0 & 1 & 0 & 0 & 2 & 1 \\
& 0 & 0 & 1 & 0 & -1 & 0 \\
& 0 & 0 & 0 & 1 & -1 & 0 \\
& 1 & 0 & 1 & 0 & 0 & 0 \\
& 0 & 0 & 0 & 1 & 0 & 0 \\
& 0 & 1 & 1 & 0 & 1 & 1 \\
& 1 & 1 & 1 & 0 & 2 & 1 \\
& 1 & 0 & 1 & 1 & -1 & 0 \\
& 1 & 1 & 0 & 1 & 2 & 1 \\
& 1 & 1 & 1 & 1 & 1 & 1 \\
\hline
\end{tabular}

for binary senior gaining-sharing stage in the following manner:

Case 1. When $f\left(x_{\text {middle }}\right)<f\left(x_{t}\right)$ : it contains three different vectors $\left(x_{\text {best }}, x_{\text {middle }}, x_{\text {worst }}\right)$, and they can assume only binary values ( 0 and 1 ), and thus a total of eight combinations are possible to update the individuals. These total eight combinations can be classified into two subcases ((a) and (b)) and each subcase contains only four different combinations. The obtained results of this case are presented in Table 8.

Subcase (a): if $x_{\text {best }}$ is equal to $x_{\text {worst }}$, then the obtained results are equal to $x_{\text {middle }}$

Subcase (b): on the other hand, if $x_{\text {best }}$ is not equal to $x_{\text {worst }}$, then the results are equal to $x_{\text {best }}$ with assuming -1 or 2 equivalent to their nearest binary value ( 0 and 1 , respectively)

Case 1 can be mathematically formulated in the following way:

$$
x_{t p}^{\text {new }}= \begin{cases}x_{\text {middle }} ; & \text { if } x_{\text {best }}=x_{\text {worst }} \\ x_{\text {best }} ; & \text { if } x_{\text {best }} \neq x_{\text {worst }} .\end{cases}
$$

Case 2. When $f\left(x_{\text {middle }}\right)<f\left(x_{t}\right)$ : it consists of four different binary vectors $\left(x_{\text {best }}, x_{\text {middle }}, x_{\text {worst }}, x_{t}\right)$, and
TABLE 8: Results of binary senior gaining and sharing stage of Case 1 with $k_{f}=1$.

\begin{tabular}{lccccc}
\hline & $x_{\text {best }}$ & $x_{\text {worst }}$ & $x_{\text {middle }}$ & Results & Modified results \\
\hline \multirow{3}{*}{ Subcase (a) } & 0 & 0 & 0 & 0 & 0 \\
& 0 & 0 & 1 & 1 & 1 \\
& 1 & 1 & 0 & 0 & 0 \\
& 1 & 1 & 1 & 1 & 1 \\
\hline \multirow{3}{*}{ Subcase (b) } & 1 & 0 & 0 & 1 & 1 \\
& 1 & 0 & 1 & 2 & 1 \\
& 0 & 1 & 0 & -1 & 0 \\
\hline
\end{tabular}

TABLE 9: Results of binary senior gaining and sharing stage of Case 2 with $k_{f}=1$.

\begin{tabular}{|c|c|c|c|c|c|c|}
\hline & $x_{\text {best }}$ & $x_{t}$ & $x_{\text {worst }}$ & $x_{\text {middle }}$ & Results & Modified results \\
\hline \multirow{4}{*}{ Subcase (c) } & 1 & 1 & 0 & 0 & 3 & 1 \\
\hline & 1 & 0 & 0 & 0 & 1 & 1 \\
\hline & 0 & 1 & 1 & 1 & 0 & 0 \\
\hline & 0 & 0 & 1 & 1 & -2 & 0 \\
\hline \multirow{12}{*}{ Subcase (d) } & 0 & 0 & 0 & 0 & 0 & 0 \\
\hline & 0 & 1 & 0 & 0 & 2 & 1 \\
\hline & 0 & 0 & 1 & 0 & -1 & 0 \\
\hline & 0 & 0 & 0 & 1 & -1 & 0 \\
\hline & 1 & 0 & 1 & 0 & 0 & 0 \\
\hline & 1 & 0 & 0 & 1 & 0 & 0 \\
\hline & 0 & 1 & 1 & 0 & 1 & 1 \\
\hline & 0 & 1 & 0 & 1 & 1 & 1 \\
\hline & 1 & 1 & 1 & 0 & 2 & 1 \\
\hline & 1 & 0 & 1 & 1 & -1 & 0 \\
\hline & 1 & 1 & 0 & 1 & 2 & 1 \\
\hline & 1 & 1 & 1 & 1 & 1 & 1 \\
\hline
\end{tabular}

with the values of each vector, a total of sixteen combinations are presented. The sixteen combinations are also divided into two subcases ((c) and (d)). Subcases (c) and (d) further contain four and twelve combinations, respectively. The subcases are explained in detail in Table 9.

Subcase (c): when $x_{\text {best }}$ is not equal to $x_{\text {worst }}$ and $x_{\text {worst }}$ is equal to $x_{\text {middle, }}$, then the obtained results are equal to $x_{\text {best }}$

Subcase (d): if any case arises other than (c), then the obtained results are equal to $x_{t}$ by taking -2 and -1 as 0 and 2 and 3 as 1

The mathematical formulation of Case 2 is given as follows:

$$
x_{t p}^{\text {new }}= \begin{cases}x_{\text {best }} ; & \text { if } x_{\text {best }} \neq x_{\text {worst }}=x_{\text {middle }} \\ x_{t} ; & \text { otherwise. }\end{cases}
$$

The pseudocode of BGSK is presented in Figure 7.

5.3. Example of Results. The STAP is solved using the proposed novel BGSK algorithm and the values of parameters are presented in Table 10. BGSK runs over personal computer Intel ${ }^{\circledR}$ CoreTM i5-7200U CPU @ $2.50 \mathrm{GHz}$ and 4 GB RAM and coded on MATLAB R2015a. To get the optimal solutions, 30 independent runs are performed. 


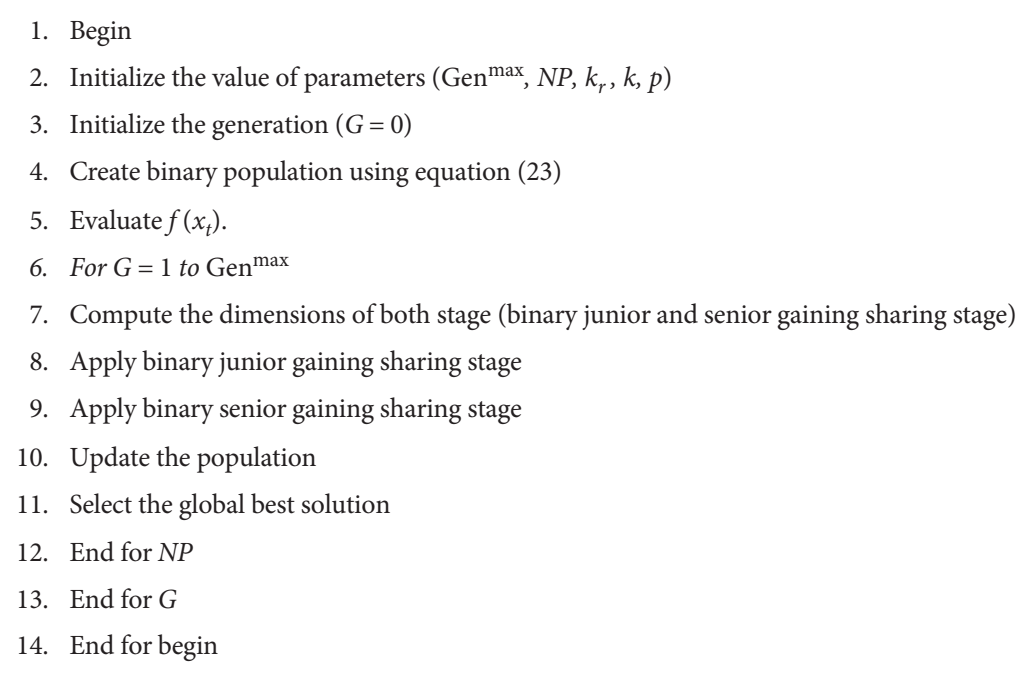

Figure 7: Pseudocode for BGSK.

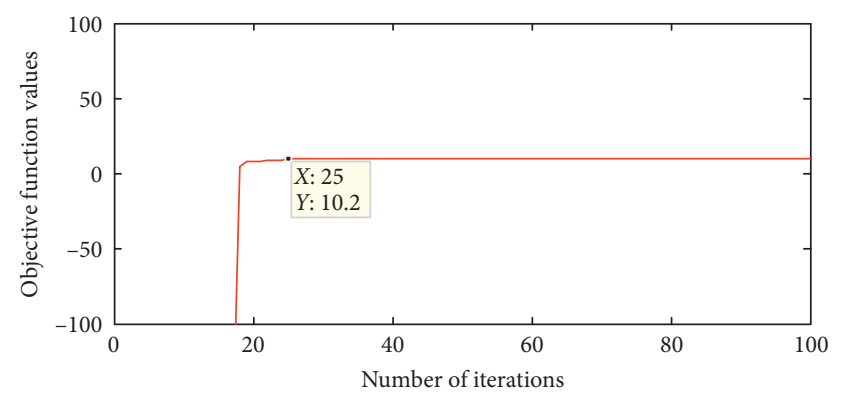

Figure 8: Convergence graph of BGSK.

Moreover, Figure 8 shows the convergence graph of the solutions of STAP using BGSK. From the figure, it can be observed that after the $25^{\text {th }}$ iteration, it converges to the global optimal solution, which shows the robustness of the BGSK.

The obtained optimum solutions for each simulation run including total profit, route length (hours), and visited workplaces are presented in Table 11. Figure 9 illustrates the histogram for the count of runs of the visited workplaces in the optimum solutions.

From Figure 9, it is resulted that visiting workplace numbers 3 and 2, respectively (Eastern Company and Eva Pharma), is the most occurring solution in $40 \%$ of the cases. The second occurring solution is to visit workplaces 3 and 5 with a chance of $20 \%$ only.

Histogram, goodness of fit, graph fitting, and descriptive statistics of the simulation results for the obtained are instructed. The histogram for the total profit and the route length is obtained. Table 12 shows the goodness-of-fit summary of the simulation results for the total profit and the route length. From the table and the shape of the PDF of the distributions, it is concluded that the goodness-of-fit test is in favor of the normal distribution for the total profit and the beta distribution for the route length.

The histograms and the best-fitted distributions for total profit and route length for the results are shown in Figure 10.
TABle 10: Numerical values of parameters.

\begin{tabular}{lc}
\hline Parameters of BGSK & Considered values \\
\hline $\mathrm{NP}$ & 200 \\
$\mathrm{k}$ & 10 \\
$k_{r}$ & 0.9 \\
$p$ & 0.1 \\
$k_{f}$ & 1 \\
Maximum number of iterations & 100 \\
\hline
\end{tabular}

TABle 11: Optimum solutions for the 30 simulation runs.

\begin{tabular}{|c|c|c|c|}
\hline$\#$ & Total profit (\$) & Route length (hours) & Visited workplaces \\
\hline 1 & 10.2 & 7.04 & 2,3 \\
\hline 2 & 10.2 & 7.74 & 5,3 \\
\hline 3 & 10.2 & 6.70 & 3,2 \\
\hline 4 & 9.00 & 7.21 & 1,2 \\
\hline 5 & 10.2 & 6.82 & 3,2 \\
\hline 6 & 9.00 & 7.01 & 3,5 \\
\hline 7 & 9.00 & 7.68 & 5,3 \\
\hline 8 & 9.00 & 6.93 & 3,5 \\
\hline 9 & 10.2 & 6.83 & 3,2 \\
\hline 10 & 10.2 & 6.71 & 3,2 \\
\hline 11 & 8.20 & 5.01 & 2,5 \\
\hline 12 & 10.2 & 7.33 & 3,2 \\
\hline 13 & 10.2 & 8.00 & 3,2 \\
\hline 14 & 8.20 & 5.29 & 5,2 \\
\hline 15 & 10.2 & 6.74 & 3,2 \\
\hline 16 & 9.00 & 7.23 & 3,5 \\
\hline 17 & 10.2 & 6.56 & 3,2 \\
\hline 18 & 9.00 & 7.11 & 3,5 \\
\hline 19 & 10.2 & 6.91 & 2,3 \\
\hline 20 & 8.60 & 7.71 & 3,1 \\
\hline 21 & 10.2 & 7.11 & 3,2 \\
\hline 22 & 10.2 & 7.17 & 2,3 \\
\hline 23 & 9.00 & 7.57 & 5,3 \\
\hline 24 & 9.00 & 7.52 & 3,5 \\
\hline 25 & 10.2 & 6.69 & 3,2 \\
\hline 26 & 10.2 & 6.81 & 3,2 \\
\hline 27 & 9.00 & 7.41 & 3,5 \\
\hline 28 & 9.00 & 7.85 & 5,3 \\
\hline 29 & 9.00 & 7.78 & 5,3 \\
\hline 30 & 10.2 & 7.43 & 3,2 \\
\hline
\end{tabular}




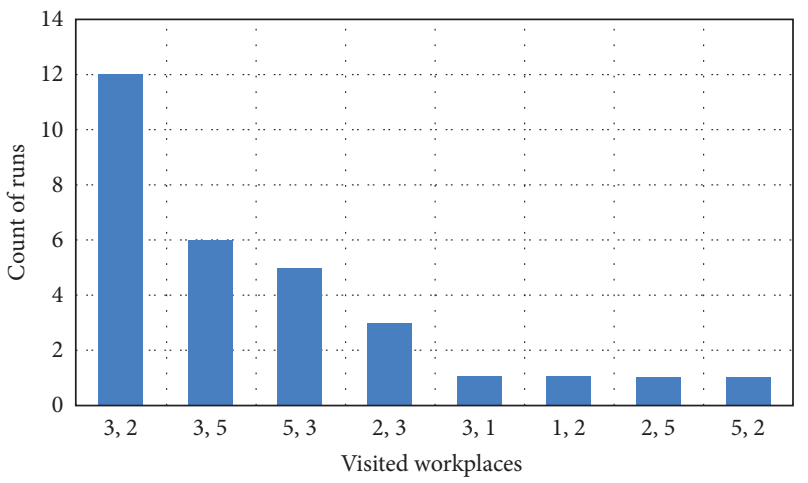

Figure 9: Histogram for the count of runs of the visited workplaces in the optimum solutions.

TABLE 12: Goodness-of-fit summary for the total profit and the route length.

\begin{tabular}{|c|c|c|c|c|c|c|}
\hline \multirow{2}{*}{$\begin{array}{l}\text { Distribution } \\
\text { Statistic } \\
\end{array}$} & \multicolumn{2}{|c|}{ Kolmogorov-Smirnov } & \multicolumn{2}{|c|}{ Anderson-Darling } & \multirow{2}{*}{\multicolumn{2}{|c|}{$\begin{array}{l}\text { Chi-squared } \\
\text { Rank }\end{array}$}} \\
\hline & Rank & Statistic & Rank & Statistic & & \\
\hline \multicolumn{7}{|l|}{ Total profit } \\
\hline Beta & 0.47633 & 3 & 11.884 & 3 & 1.0222 & 1 \\
\hline Normal & 0.34462 & 2 & 3.7465 & 1 & 2.1289 & 2 \\
\hline Pert & 0.53333 & 4 & 171.24 & 4 & 10.19 & 3 \\
\hline Uniform & 0.28813 & 1 & 9.8021 & 2 & & \\
\hline Triangular & \multicolumn{6}{|c|}{ No fit } \\
\hline \multicolumn{7}{|l|}{ Route length } \\
\hline Beta & 0.14288 & 1 & 0.52661 & 1 & 0.60354 & 2 \\
\hline Normal & 0.18449 & 3 & 1.0842 & 3 & 0.19006 & 1 \\
\hline Pert & 0.17218 & 2 & 0.75239 & 2 & 1.0559 & 3 \\
\hline Triangular & 0.26604 & 5 & 1.6744 & 4 & 5.0607 & 4 \\
\hline Uniform & 0.23558 & 4 & 8.9251 & 5 & \multicolumn{2}{|c|}{ N/A } \\
\hline
\end{tabular}

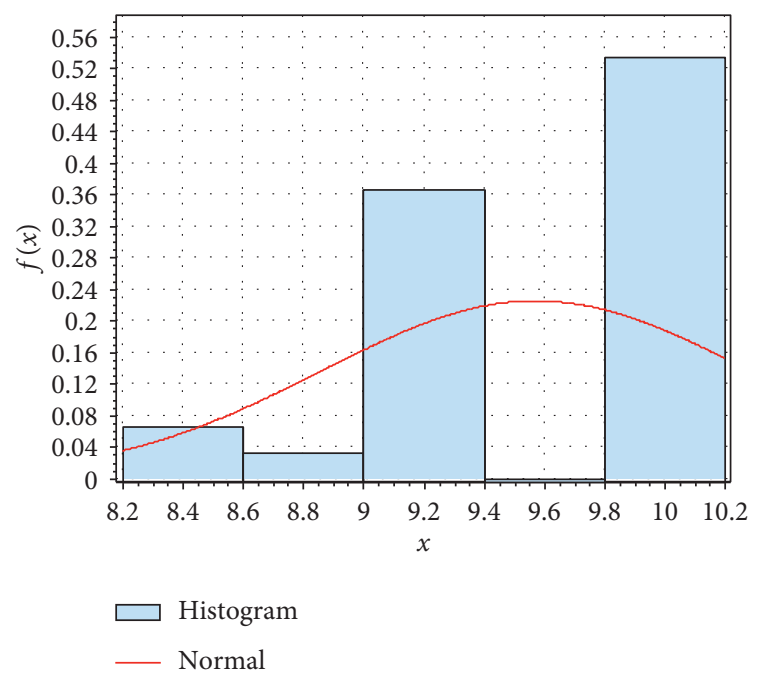

(a)

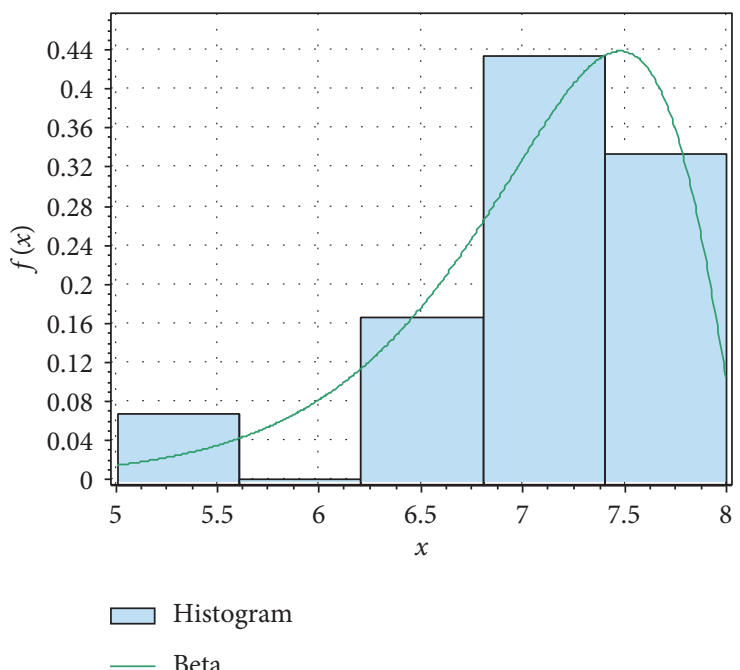

(b)

Figure 10: Histogram and distribution fitting. (a) Total profit (\$) and (b) route length (hours).

A normal distribution is the most suitable to fit the total profit with a mean of $\$ 957.33$, a minimum of $\$ 820$, and a maximum of $\$ 1020$. A beta distribution is the most suitable to fit the route length with a mean of 7.0633 , a minimum of 5.01 , and a maximum of 8 in hours. The descriptive statistics for both distributions are presented in Table 13. 
TABle 13: Descriptive statistics for the total profit and route length.

\begin{tabular}{lcc}
\hline Statistic & Total profit & Route length \\
\hline Distribution & Normal & Beta \\
Sample size & 30 & 30 \\
Range & 2 & 2.99 \\
Mean & 9.5733 & 7.0633 \\
Variance & 0.50409 & 0.42964 \\
Std. deviation & 0.70999 & 0.65547 \\
Coef. of variation & 0.07416 & 0.0928 \\
Std. error & 0.12963 & 0.11967 \\
Skewness & -0.43748 & -1.5419 \\
\hline
\end{tabular}

\section{Conclusions and Points for Future Research Studies}

The main conclusions for this paper can be summarized as follows:

(1) A new application problem called Stochastic Travelling Advisor Problem (STAP), where an advisor is likely to select the most profitable route for visiting a subset of candidate workplaces. Continuous probability distributions are used to model the stochastic variables for the problem in order to be a closer representation of real-world applications.

(2) The stochastic nature appears in the presentation of the travelling and the advising times. The simulation procedure is used to generate stochastic parameters following PERT-beta distribution and then gather results obtained from the repeated simulation runs.

(3) The problem arises extensively in the advising domain for the service sector. It differs from the deterministic version in the fact that the travelling and advising times are stochastic in nature which is the case in real-life problems.

(4) Travelling Advisor Problem (TDP) looks like the famous Travelling Salesman Problem (TSP) but with basic distinct differences, mainly, the limited available route time, visiting only a subset of the places, considering the time consumed in customer places as a basic component, and the objective of maximizing the total profit.

(5) A nonlinear binary mathematical model is formulated for the given stochastic problem. The binary decision variables represent the allocation of chosen workplaces into the positions of the proposed route, and the nonlinearity appears in the limited time constraint. The final objective is to maximize the total profit of the chosen route.

(6) A real application case study in the field of occupational health and safety as one of the very important fields of the service sectors is presented. The stochastic model is formulated and the simulation run problems are solved, and the output histograms and the best-fitted distributions for the total profit and for the route length are obtained.

(7) To obtain the solution of the proposed STAP nonlinear binary programming model, a novel binary gaining-sharing knowledge-based optimization algorithm (BGSK) is introduced which includes the two main binary junior and senior gaining-sharing stages with the knowledge factor $k_{f}=1$. The BGSK is the first variant of the developed GSK, and the proposed algorithm is applied with an augmented Lagrangian method to handle the constraints.

(8) The nonlinear binary mathematical model and the solution method are used to mimic and solve a real case study example. The optimal solution is obtained which is more profitable by $10.8 \%$ than the solution given by the management based on selecting workplaces with the highest profit under the time limit constraint.

(9) The obtained results by BGSK show its robustness and convergence and prove that it can find the global optimal solution of STAP. BGSK gives better results when compared to the health and safety agency management.

The points for future research studies can be stated as follows:

(1) To propose other stochastic mathematical models' formulations for the same problem starting with the design of the decision variables and compare the effectiveness of computations for each model

(2) To augment the proposed Stochastic Travelling Advisor Problem (STAP) with its variations as STAP with time window (STAPTW), STAP with multiple advisors (mSTAP), Multiobjective Stochastic Multiple Travelling Advisor Problem (MOSmTAP), and other variations.

(3) To apply the same problem formulation to other similar advisory fields that can show up in many other consulting domains such as industry, agriculture, business, 
education, telecommunications, investing, quality assurance, social and community services, pollution, medical, tourism, marketing, sales, advertising, sports, arts, and cooking.

(4) To check the performance of the BGSK algorithm in solving different types of complex optimization problems, and further works can be investigated by the extension of BGSK.

\section{Data Availability}

Actually, data are available on request. The interested researchers can contact the corresponding author.

\section{Conflicts of Interest}

The authors declare that they have no conflicts of interest.

\section{Acknowledgments}

This research was funded by Research Supporting Program at King Saud University, Riyadh, Saudi Arabia.

\section{References}

[1] I. Droste, "Algorithms for the travelling salesman problem," Bachelor Thesis, Utrecht University, Utrecht, Netherlands, 2017.

[2] C. C. Pinter, A Book of Set Theory, Dover Publications Inc. New York, NY, USA, 2014.

[3] D. Tas, N. P. Dellaert, and de, A. G. Kok, "Vehicle routing problem with stochastic travel times including soft time windows and service costs," Computers \& Operations Research, vol. 40, no. 1, pp. 214-224, 2013.

[4] J. Fente, K. Knutson, and C. Schexnayder, "Defining a beta distribution function for construction simulation," in Proceedings of the 31st Conference on Winter Simulation: Simulation-a Bridge to the Future, vol. 2, pp. 1010-1015, Phoenix, AZ, USA, 1999.

[5] W. Tian, C. Huang, and X. Wang, "February. A near optimal approach for symmetric traveling salesman problem in euclidean space," in Proceedings of the 6th International Conference on Operations Research and Enterprise Systems (ICORES 2017), pp. 281-287, Porto, Portugal, 2017.

[6] J. Mun, Advanced Analytical Models: Over 800 Models And 300 Applications From The Basel Ii Accord To Wall Street And Beyond, vol. 419, pp. 899-917, John Wiley \& Son, Hoboken, NJ, USA, 2008.

[7] N. L. Johnson, S. I. Kotz, and N. Balakrishnan, "Beta distributions," in Continuous univariate distributions, vol. 1, pp. 221-235, John Wiley \& Sons, New York, NY, 1994.

[8] C. Forbes, M. Evans, N. Hastings, and B. Peacock, Statistical Distributions, John Wiley \& Sons, Hoboken, NJ, USAnot, 2011.

[9] M. Y. Sulaiman, W. H. Oo, M. A. Wahab, and A. Zakaria, "Application of beta distribution model to Malaysian sunshine data," Renewable Energy, vol. 18, no. 4, pp. 573-579, 1999.

[10] P. Rosenmai, "Eureka statistics, beta distribution pdf grapher," 2015, http://eurekastatistics.com/beta-distributionpdf-grapher/.
[11] R. Nafkha, "The PERT method in estimating project duration," Information Systems in Management, vol. 5, no. 4, pp. 542-550, 2016.

[12] E. Elbeltagi, Lecture Notes on Construction Planning and Scheduling, Structural Engineering Department, Faculty of Engineering, Mansoura University, Mansoura, Egypt, 2012.

[13] P. Ballesteros-Pérez, "M-PERT: manual project-duration estimation technique for teaching scheduling basics, technical paper," Journal of Construction Engineering and Management, vol. 143, no. 9, 2017.

[14] N. R. Shankar, K. S. N. Rao, and V. Sireesha, "Estimating the mean and variance of activity duration in PERT," International Mathematical Forum, vol. 5, no. 18, pp. 861-868, 2010.

[15] H. Golpîra, "Estimating duration of projects manual tasks using MODAPTS plus method," International Journal of Research in Industrial Engineering, vol. 2, pp. 12-19, 2013.

[16] N. R. Shankar, S. S. Babu, Y. L. P. Thorani, and D. Raghuram, "Right skewed distribution of activity times in PERT," International Journal of Engineering Science and Technology (IJEST), vol. 3, no. 4, pp. 2932-2938, 2011.

[17] EasyFit, "Distribution fitting software, mathwave technologies," 2020, https://qpdownload.com/easyfit/.

[18] R. Davis, Stochastic Project Duration Analysis Using PERTBeta Distributions, San José State University, San Jose, CA, USA, 2006, https://www.sjsu.edu/cob/docs/I-06-005.pdf.

[19] R. Davis, "Teaching note-teaching project simulation in Excel using PERT-BetaDistributions," INFORMS Transactions on Education, vol. 8, no. 3, pp. 139-148, 2008.

[20] S. M. Salim, F. I. Romli, J. Besar, and N. O. Aminian, "A study on potential physical hazards at construction sites," Journal of Mechanical Engineering, vol. 4, no. 1, pp. 207-222, 2017.

[21] O. G. Bhusnure, "Chemical hazards and safety management in pharmaceutical industry," Journal of Pharmacy Research, vol. 12, no. 03, p. 2018, 2018.

[22] E. Sacadura-Leite, L. Mendonça-Galaio, O. Shapovalova, I. Pereira, R. Rocha, and A. Sousa-Uva, "Biological hazards for healthcare workers: occupational exposure to vancomycinresistant," Portuguese Journal of Public Health, vol. 36, pp. 26-31, 2018.

[23] M. Carnell and D. Nebosh, Preventing Harm in the Workplace Workbook, Milton Keynes University Hospital, NHS Foundation Trust, Eaglestone, UK, 2017, https://www. mkuhworkforce.co.uk/sites/default/files/2017-12/Health\%2C $\% 20$ Safety $\% 20 \% 26 \% 20$ Welfare.pdf.

[24] C. Coady, D.A. Feltham-Scott, C. Fewer, C. Osborne, and K. Taylor, Building a Safer Tomorrow, Workplace Health, Safety and Compensation Commission of Newfoundland and Labrador, WorkplaceNL), San Jose, CA, USA, 2015.

[25] M. S. G. Awad and A. A. Nour El-Din, "Egyptian law regulations in occupational safety and health," 2018.

[26] International Labour Organization website, (2020), https://www. ilo.org/dyn/natlex/natlex4.detail?p_lang=en\&p_isn=64693.

[27] Sika Group, "Occupational safety and health, sika egypt website,” 2020, https://egy.sika.com/content/egypt/main/ en/group/Aboutus/sustainability/environment_and_safety/ Safety.html.

[28] L. Parker, The Early Years Health and Safety Handbook, Taylor and Francis Group, London, UK, 2012.

[29] Marriott, M. Cosman, and R. Schmidt-McCleave, Safeguard Health and Safety Handbook 2019, Thomson Reuters New Zealand Limited, Wellington, New Zealand, 2018.

[30] p P. Manu, F. Emuzep, T. A. Saurin, H. W. p Bonaventura, and Hadikusumo, Construction Health and Safety in Developing Countries, Taylor and Francis Group, London, UK, 2020. 
[31] M. Bognar, "Probability distribution applets," 2020, https:// homepage.divms.uiowa.edu/\%7Embognar/.

[32] A. W. Mohamed, A. A. Hadi, and A. K. Mohamed, "Gainingsharing knowledge based algorithm for solving optimization problems: a novel nature-inspired algorithm," International Journal of Machine Learning and Cybernetics, vol. 11, no. 7, pp. 1501-1529, 2020.

[33] K. Deb, "An efficient constraint handling method for genetic algorithms," Computer Methods in Applied Mechanics and Engineering, vol. 186, no. 2-4, pp. 311-338, 2000.

[34] C. A. C. Coello, "Theoretical and numerical constrainthandling techniques used with evolutionary algorithms: a survey of the state of the art," Computer Methods in Applied Mechanics and Engineering, vol. 191, no. 11-12, pp. 1245-1287, 2002.

[35] N. Muangkote, L. Photong, and A. Sukprasert, "Effectiveness of constrained handling techniques of improved constrained differential evolution algorithm applied to constrained optimization problems in mechanical engineering," ITMSOC Transactions on Innovation \& Business Engineering, vol. 4, pp. 1-21, 2019.

[36] W. Long, X. Liang, Y. Huang, and Y. Chen, "A hybrid differential evolution augmented lagrangian method for constrained numerical and engineering optimization," ComputerAided Design, vol. 45, no. 12, pp. 1562-1574, 2013.

[37] A. Bahreininejad, "Improving the performance of water cycle algorithm using augmented Lagrangian method," Advances in Engineering Software, vol. 132, pp. 55-64, 2019. 